\title{
Effectiveness of medical thoracoscopy and thoracoscopic talc poudrage in patients with exudative pleural effusion
}

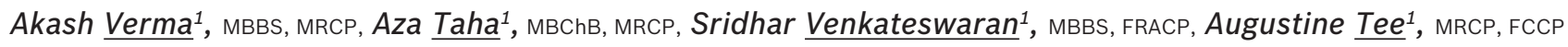

INTRODUCTION This study aimed to assess the effectiveness of medical thoracoscopy (MT) and thoracoscopic talc poudrage (TTP) in patients with exudative pleural effusion.

METHODS We evaluated the diagnostic yields, complications and outcomes of MT and TTP in 41 consecutive patients with symptomatic pleural effusions who were planned to undergo both procedures from 1 December 2011 to 30 November 2012. Data was reviewed retrospectively and prospectively up to March 2013.

RESULTS Among the 41 patients, 36 underwent MT with the intent of biopsy and talc pleurodesis, 2 underwent MT for pleurodesis only and 3 had failed MT. Aetiologies of pleural effusion included lung cancer $(n=14)$, tuberculosis $(n=9)$, breast cancer $(n=7)$, ovarian cancer $(n=2)$, malignant mesothelioma $(n=1)$, congestive cardiac failure $(n=1)$, peritoneal dialysis $(n=1)$ and hepatic hydrothorax $(n=1)$; pleural effusion was undiagnosed in five patients. The overall diagnostic yield of MT, and the yield in tubercular and malignant pleural effusions were $77.8 \%, 100.0 \%$ and $82.6 \%$, respectively; it was inconclusive in $22.2 \%$. Complications that occurred were self-limiting, with no procedure-related mortality. The 30-day mortality rate was $17.1 \%$. A total of 15 patients underwent TTP. The 30-, 60- and 90 -day success rates were $77.8 \%$, $80.0 \%$ and $80.0 \%$, respectively, with one patient having complications (i.e. empyema). The 30 -day mortality was $40.0 \%$. CONCLUSION MT is a safe procedure with high diagnostic yields in undiagnosed pleural effusions. TTP is an effective method to stop recurrence of pleural effusions.

Keywords: chest tube, medical thoracoscopy, pleural effusion, pleurodesis, pleuroscopy

\section{INTRODUCTION}

Medical thoracoscopy (MT) is most commonly indicated to establish a diagnosis of indeterminate pleural effusion and to enable pleurodesis to be performed safely and effectively. ${ }^{(1)}$ Approximately $25 \%$ of all pleural abnormalities remain undiagnosed after thoracentesis and/or closed pleural biopsies. ${ }^{(2-4)}$ In malignant and tuberculous effusions, the respective diagnostic yields for the following procedures are as follows: pleural fluid analysis alone $-62 \%$ and $28 \%$; closed pleural biopsy alone $-44 \%$ and $51 \%$; and a combination of the two procedures $-74 \%$ and $61 \% .{ }^{(1)}$ The diagnostic yields for MT in malignant and tuberculous effusions are $95 \%$ and $99 \%$, respectively. ${ }^{(1)}$

Pleurodesis is the preferred treatment for recurrent malignant effusion or benign effusion, and the use of talc is preferred over other sclerosing agents (e.g. mitozantrone and tetracycline). The recommended technique of administration of talc for pleurodesis is thoracoscopic talc poudrage (TTP) instead of talc slurry instillation. ${ }^{(5)}$ The purpose of the present study was to assess the effectiveness of MT in establishing the diagnosis of indeterminate pleural effusion, and enabling pleurodesis to be performed safely and effectively in 41 patients with exudative pleural effusion.

\section{METHODS}

A total of 41 consecutive patients with unilateral symptomatic pleural effusion, who underwent MT at our centre between 1 December 2011 and 30 November 2012 and received follow-up until March 2013, were included in the present study. The clinical characteristics of these patients are presented in Table I. Chest radiography and thoracic ultrasonography were performed to determine the extent of pleural effusion. Prior to MT, thoracentesis was performed for cytopathological and microbiological analyses.

All MTs were performed by a pulmonary physician who was assisted by two trained nurses in an endoscopy suite. The patients were placed in the lateral decubitus position for the MT. The patients' blood pressure, pulse rate and oxygen saturation were monitored continuously throughout the procedure. Supplemental oxygen was given to the patients to maintain oxygen saturation. Lidocaine $2 \%$ was used for local anaesthesia, and sedation was achieved using a combination of midazolam and fentanyl. We used a 7.0-mm Evis Exera Pleuravideoscope (Olympus LTF-160; Olympus, Tokyo, Japan) with a single 8-mm trocar. After complete aspiration of the remaining fluid was done, a thorough inspection of the pleural surface was performed. Adhesions were taken down with biopsy forceps, where possible. Biopsy specimens were sent for histopathology examination. Under visual control, an average of $6 \mathrm{~g}$ of sterile asbestos-free talc (Steritalc; Novatech, La Ciotat, France) was distributed onto the pleural surface of patients who were judged to have expandable lung based on thoracoscopic findings. After the thoracoscope was removed, a thoracostomy tube (20-French) was inserted. Suction $\left(2 \mathrm{Cm} \mathrm{H}_{2} \mathrm{O}\right)$ was started after one hour; the chest tube was left in place until $\leq 150 \mathrm{~mL}$ of

${ }^{1}$ Department of Respiratory and Critical Care Medicine, Changi General Hospital, Singapore

Correspondence: Dr Aza Taha, Resident, Department of Respiratory and Critical Care Medicine, Changi General Hospital, 2 Simei Street 3 , Singapore 529889. azataha75@gmail.com 
Table I. Clinical characteristics of the patients $(n=41)$.

\begin{tabular}{|c|c|}
\hline Characteristic & No. (\%) \\
\hline 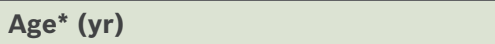 & $65 \pm 15$ \\
\hline \multicolumn{2}{|l|}{ Gender } \\
\hline Male & $22(53.7)$ \\
\hline Female & $19(46.3)$ \\
\hline \multicolumn{2}{|l|}{ Ethnicity } \\
\hline Chinese & $26(63.4)$ \\
\hline Malay & $11(26.8)$ \\
\hline Indian & $4(9.8)$ \\
\hline \multicolumn{2}{|l|}{ Final diagnosis } \\
\hline Malignant & $24(58.5)$ \\
\hline NSCLC & $13(31.7)$ \\
\hline Malignant mesothelioma & $1(2.4)$ \\
\hline Sarcomatoid carcinoma of the lung & $1(2.4)$ \\
\hline Breast cancer & $7(17.1)$ \\
\hline Ovarian cancer & $2(4.9)$ \\
\hline Benign & $12(29.3)$ \\
\hline Tuberculosis & $9(22.0)$ \\
\hline Others ${ }^{+}$ & $3(7.3)$ \\
\hline Undiagnosed & $5(12.2)$ \\
\hline
\end{tabular}

*Data presented as mean \pm standard deviation. †Includes congestive cardiac failure, hepatic hydrothorax and peritoneal dialysis-induced pleural effusion. NSCLC: non-small cell lung cancer

fluid was drained in 24 hours. Chest radiography was performed on the same day after the procedure and before discharge. Tumour type, initial response, duration of suction treatment, side effects and procedure-related complications were documented.

In the present study, diagnostic success of MT was defined as the demonstration of (a) malignant cells in cases of malignancy; (b) granuloma in cases of tuberculosis (TB); and (c) other findings that point toward a conclusive diagnosis for other diseases. Therapeutic success (i.e. success of pleurodesis) was defined as an absence of fluid accumulation with long-term symptom relief. All cases with recurrent symptomatic effusion that required drainage were deemed as failures, in accordance with the recommendations of the American Thoracic Society. ${ }^{(6)}$ Success and survival rates were calculated with the day of the procedure taken as the start date. Follow-up information was obtained via the records of periodic clinical examinations and chest radiography conducted in our outpatient clinic. In addition, hospice care centres were contacted for supplementary outcome information. The primary end point was symptomatic recurrence of pleural fluid that required drainage.

Data was analysed using the Statistical Package for the Social Sciences version 19 (SPSS Inc, Chicago, IL, USA). The clinical features were compared against the outcomes using Wilcoxon two-sample test or Fisher's exact test. Two-sided p-values were used, and a p-value $<0.05$ was considered indicative of a significant difference.

\section{RESULTS}

A total of 41 patients underwent MT between 1 December 2011 and 30 November 2012. The indications were: (a) undiagnosed effusions after thoracentesis and closed biopsy $(\mathrm{n}=25)$; (b) biopsy and TTP in a single sitting ( $\mathrm{n}=13)$; (c) TTP only $(\mathrm{n}=2)$; and (d) tissue biopsy for epidermal growth factor receptor and anaplastic lymphoma kinase mutation analysis when the tissue provided by closed biopsy was considered inadequate $(n=1)$. Among the 41 patients, biopsies were planned for 39 patients; however, only 36 underwent biopsy (Fig. 1). The clinical characteristics and final diagnoses of the patients are shown in Table I. The diagnostic yield of MT was $77.8 \%$ (28/36) in all cases of exudative pleural effusion, $100.0 \%$ (8/8) in cases of tubercular pleural effusion and $82.6 \%(19 / 23)$ in cases of malignant pleural effusion (Table II). Thoracoscopic biopsy was non-diagnostic in $8(22.2 \%)$ patients. Among these eight patients, four had a diagnosis of sarcomatoid lung cancer $(n=1)$, breast cancer ( $n=1)$ and ovarian cancer $(n=2)$, which were obtained by bronchoscopy, breast biopsy and ovarian biopsy, respectively. A diagnosis was not established for the latter four patients, even up to the end of the follow-up period (i.e. March 2013), and TB was excluded for these patients as their tissue cultures were negative for TB. Out of these four undiagnosed patients, one died; the other three patients remained well at four months follow-up, indicating the likelihood that the disease was of benign aetiology.

MT failed in 3 of the 41 (7.3\%) patients, as the scope could not be passed into the pleural cavity secondary to minimal residual effusion and expanded lung (due to prior drainage). No biopsy could be performed in these three patients. Of the three patients, two were eventually diagnosed with lung cancer and TB (via endobronchial ultrasonography-guided transbronchial needle aspiration and bronchoscopy, respectively), while the other patient remained undiagnosed. Complications occurred in $6(14.6 \%)$ of the patients, and these included subcutaneous emphysema $(n=2)$, pneumothorax $(n=3)$ and hypoxaemia $(n=1)$. The complications were minor and self-limiting in all except the hypoxic patient, who required intubation and intensive care unit monitoring for 48 hours. The 30-day mortality was $17.1 \%(n=7)$, while the 90-day mortality was $39.0 \%(n=16)$. There was no procedure-related mortality.

A total of 15 patients underwent TTP. Of these, 13 patients had malignant disease, and the main tumour types were lung cancer $(n=7)$ and breast cancer $(n=6)$. Two patients had benign conditions, namely hepatic hydrothorax $(n=1)$ and recurrent effusion from peritoneal dialysis $(n=1)$. TTP was indicated in patients with benign disease if they had multiple episodes of recurrence and refractoriness to primary therapy. The median duration of suction treatment was 4.5 (range 1-6) days. There were no procedure-related deaths. The 30 -day mortality rate was $40.0 \%(n=6)$; five of these six patients died from rapid progression of end-stage malignant disease and one from a fall, sustaining intracranial haemorrhage contributed by thrombocytopenia secondary to alcoholic liver disease. The patient who had intracranial haemorrhage following a fall developed empyema after thoracoscopy for hepatic hydrothorax, and the empyema was successfully treated with antibiotics. Of the five patients who died from malignant disease, two had breast carcinoma, two had non-small cell lung cancer and one had sarcomatoid carcinoma of the lung. Thus, only 9 of the 15 patients who underwent TTP were 


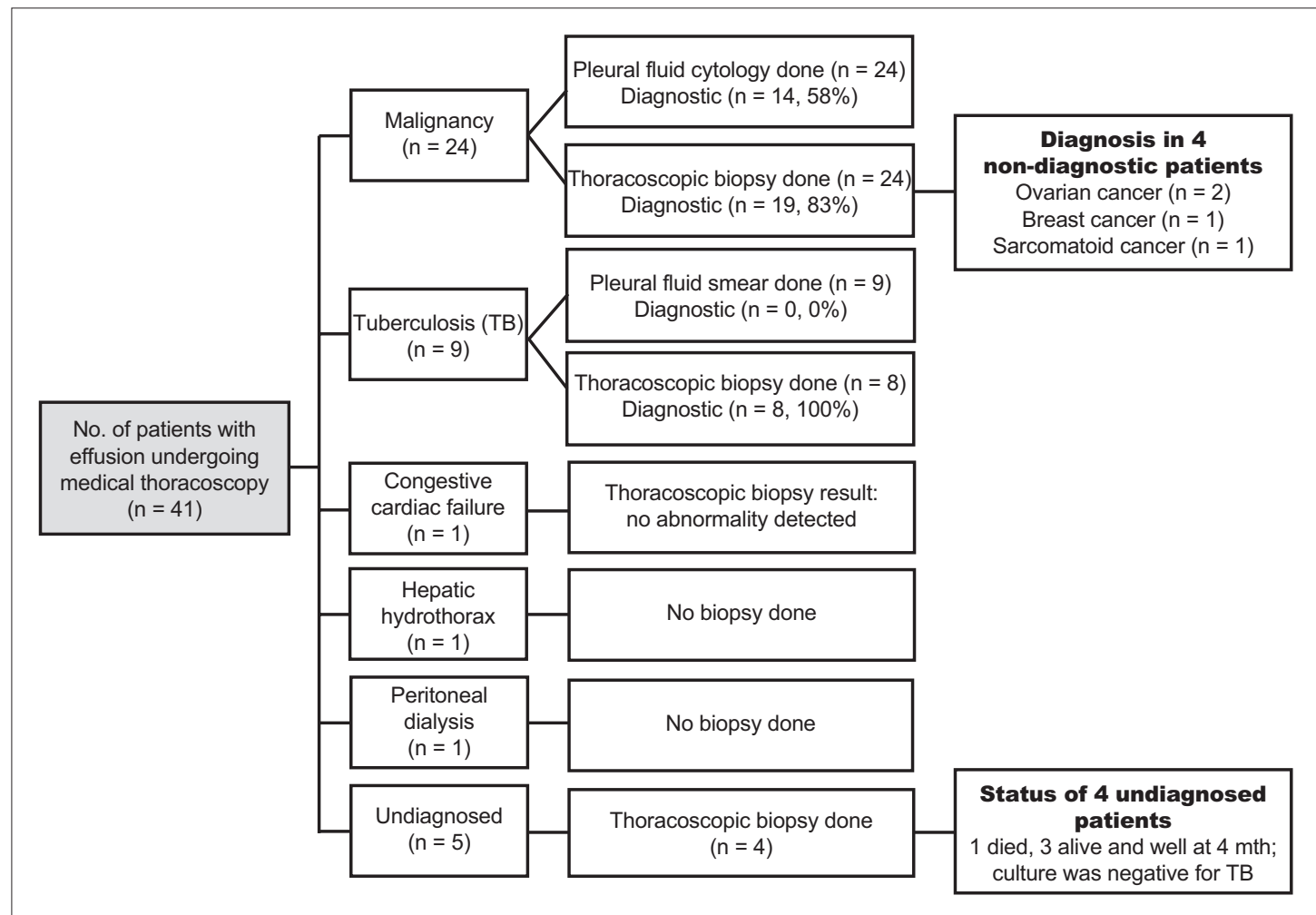

Fig. 1 Flow chart shows the results of medical thoracoscopy.

Table II. Diagnostic yield of the various diagnostic tests.

\begin{tabular}{lc}
\hline Diagnostic test & $\begin{array}{c}\text { No. }(\%) \text { of } \\
\text { positive results }\end{array}$ \\
\hline Thoracoscopic biopsy $(\mathbf{n}=\mathbf{3 6})$ & $28(77.8)$ \\
Final diagnosis of malignancy $(\mathbf{n}=\mathbf{2 4})$ & $19(82.6)^{\star}$ \\
$\quad \begin{array}{l}\text { Thoracoscopic pleural biopsy }(\mathrm{n}=23) \\
\text { Pleural fluid cytology }(\mathrm{n}=24)\end{array}$ & $14(58.3)$ \\
Final diagnosis of tuberculosis $(\mathbf{n}=\mathbf{9})$ & $8(100.0)^{+}$ \\
Thoracoscopic pleural biopsy showing & \\
granuloma $(\mathrm{n}=8)$ & $0(0)$ \\
$\begin{array}{l}\text { Pleural fluid smear positive for AFB }(n=9) \\
\text { Sputum smear positive for AFB }(n=9)\end{array}$ & $0(0)$ \\
Bronchoscopic alveolar lavage positive for & $1(100)$ \\
AFB $(n=1)$ & \\
\hline
\end{tabular}

*One patient was excluded from the analysis, as thoracoscopic biopsy could not be performed; the diagnosis for this patient was established by endobronchial ultrasonography-guided transbronchial needle aspiration. †One patient was excluded from the analysis, as thoracoscopic biopsy could not be performed; the diagnosis for this patient was established by bronchoscopic alveolar lavage. AFB: acid-fast bacteria

evaluable at one month. Out of these nine patients, $7(77.8 \%)$ had positive responses with complete effusion control. Tumour type and pleural fluid $\mathrm{pH}$ were found to have no significant influence on the success rate of TTP $(p=0.19$ and $p=0.98$, respectively; Table III).

The 30-day, 60-day and 90-day success rates were $77.8 \%$, $80.0 \%$ and $80.0 \%$, respectively (Table IV). The median duration of follow-up ( $\mathrm{n}=5$ ) was 124 (range 101-425) days. Overall, 12 patients had lasting pleural symphysis until death or the date of the last follow-up. The 30-day mortality among the 15 patients who underwent TTP was $40.0 \%(n=6)$, while the 90-day mortality was $66.7 \%(n=10)$ (Table V). Response to pleurodesis did not
Table III. Relationship between the success rates of thoracoscopic talc poudrage and tumour type and fluid $\mathrm{pH}$.

\begin{tabular}{lccc}
\hline Variable & Success $(\mathbf{n}=\mathbf{1 2})^{*}$ & Failure $(\mathbf{n}=\mathbf{3})^{*}$ & p-value \\
\hline Fluid pH & $7.62(7.13-8)$ & $7.85(7.34-7.85)$ & 0.98 \\
$\begin{array}{l}\text { Duration of chest } \\
\text { tube usage (days) }\end{array}$ & $4(2-6)$ & $5(4-5)$ & 0.82 \\
Breast cancer & $6(50)$ & 0 & - \\
Lung cancer & $4(33.3)$ & $3(100)$ & 0.19 \\
\hline
\end{tabular}

*Data presented as either median (range) or no. (\%)

Table IV. Success rate of pleurodesis in the 15 patients who underwent thoracoscopic talc poudrage.

\begin{tabular}{lc}
\hline $\begin{array}{l}\text { Time after } \\
\text { pleurodesis (day) }\end{array}$ & $\begin{array}{c}\text { No. of patients with successful } \\
\text { pleurodesis/no. of patients alive (\%) }\end{array}$ \\
\hline 30 & $7 / 9(77.8)$ \\
60 & $4 / 5(80.0)$ \\
90 & $4 / 5(80.0)$ \\
\hline
\end{tabular}

Table V. Survival rate of the 15 patients who underwent thoracoscopic talc poudrage.

\begin{tabular}{lc}
\hline $\begin{array}{l}\text { Time after } \\
\text { pleurodesis (day) }\end{array}$ & $\begin{array}{c}\text { No. of patients alive/total } \\
\text { no. of patients (\%) }\end{array}$ \\
\hline 30 & $9 / 15(60.0)$ \\
60 & $5 / 15(33.3)$ \\
90 & $5 / 15(33.3)$ \\
120 & $4 / 15(26.7)$ \\
150 & $1 / 15(6.7)^{*}$ \\
\hline
\end{tabular}

*All but one patient who had pleural effusion secondary to a benign condition (i.e. chronic renal failure and peritoneal dialysis-induced pleural effusion) died by 150 days. 
predict survival at 30 days $(p=0.8)$ and survival at 90 days $(p=0.73)$. The median durations of survival for breast cancer and lung cancer were 49 (range 15-123) days and 24 (17-35) days, respectively $(p=0.31)$.

\section{DISCUSSION}

Approximately $25 \%$ of all pleural abnormalities remain undiagnosed after thoracentesis and/or closed pleural biopsies. ${ }^{(2-4)}$ In patients with undiagnosed pleural effusion, MT is recommended. This is because MT has a diagnostic yield of $95 \%$ for malignant effusion and a diagnostic yield of $99 \%$ for tuberculous effusion. ${ }^{(1)}$ TTP is recommended for malignant pleural effusions and recurrent benign effusions. Although it is uncertain whether the technique for administering talc affects the outcome, a meta-analysis showed that thoracoscopic insufflation of talc was more effective in achieving non-recurrence of the effusion as compared to bedside instillation of talc slurry through a chest tube (relative risk 1.19, 95\% confidence interval 1.04-1.36). ${ }^{(5)}$ However, a randomised multicentre trial of 482 patients reported that the two aforementioned techniques for administering talc had equal efficacy at 30 days (78\% vs. $71 \%){ }^{.7)}$ The purpose of the present study was to evaluate the effectiveness of MT in: (a) the diagnosis of indeterminate pleural effusion; and (b) pleurodesis by talc insufflation.

In the present study, MT yielded a diagnosis in about $78 \%$ of the patients. This rate of diagnostic yield is consistent with those reported in other studies (i.e. $74.3 \%-96.0 \%$ ), but the overall yield found in our study is lower. ${ }^{(8-11)}$ However, the diagnostic yields for cases of malignant and tubercular pleural effusions $(82.6 \%$ and $100.0 \%$, respectively) in our study are superior to the published combined yields of pleural fluid cytology and needle biopsy in malignant and tubercular pleural effusions (74\% and $61 \%$, respectively). ${ }^{(1)}$

Among the eight patients with non-diagnostic MT in the present study, the diagnoses of four patients were obtained from sites other than the pleura. Three of these four patients were eventually diagnosed with ovarian cancer via ovarian biopsy $(\mathrm{n}=2)$ and breast carcinoma via a breast nodule biopsy $(\mathrm{n}=1)$. No visible pleural nodules or abnormality was detected in these patients. As there was no target lesion to guide the biopsies in these three patients, the biopsies were taken randomly, which may account for why the thoracoscopic biopsy results were negative. It can be envisaged that in the absence of visible pleural abnormality, the yield of thoracoscopic biopsy may drop. The remaining patient was a 77-year-old man with several masses in his lung, along with left pleural effusion; pleural effusion cytology was negative. Together with the speculated mass in the right lower lobe, this patient had a mass centred at the pedicle of the fourth thoracic vertebra and multiple destructive bony lesions suspicious of metastatic disease involving the thoracic and lumbar vertebrae. There was a left paraspinal soft tissue mass invading the left neural exit foramina and the spinal canal, with mild cord compression. Diagnosis in this patient was obtained via bronchoscopic biopsy of the right lower lobe mass, which showed sarcomatoid carcinoma of lung, with immunostains that were negative for thyroid transcription factor- 1 and p63 (markers of adenocarcinoma of lung origin and squamous cell carcinoma, respectively). ${ }^{(12)}$ The negative immunostains suggested malignancy of non-pulmonary origin. This patient died 17 days after the procedure. It remains unclear whether he had primary sarcomatoid carcinoma of the lung or pulmonary metastasis from a sarcoma of non-pulmonary origin. The development of metastasis from sarcomas of non-pulmonary origin carries a poor prognosis. In such metastatic disease, $62 \%-80 \%$ of patients would have pulmonary metastases and 50\%-70\% isolated pulmonary metastases. ${ }^{(13)}$ The most common metastatic route for soft tissue sarcomas of the extremities is via the venous system. ${ }^{(13)}$ Pleural effusions do not develop in cases of pleura involvement by secondary sarcoma because of the absence of lymphatic metastases. ${ }^{(14)}$ Hence, the effusion in this patient can be considered as paramalignant rather than malignant, thus explaining the negative thoracoscopic biopsy. However, there is limited published data on primary sarcomatoid carcinoma of the lung and no data on the yield of pleural biopsy for this type of cancer. Thus, it is difficult to comment on whether the negative thoracoscopic biopsy in the patient was a false negative or a true negative secondary to a lack of malignant involvement of the pleura.

In the present study, complications from MT were minor and self-limiting, with no periprocedural mortality noted. Although one patient needed intubation 12 hours after thoracoscopy, monitoring in the intensive care unit for 48 hours and a second chest drain to drain the postprocedural pneumothorax, the patient subsequently recovered and survived for 30 days.

It has been reported that $7 \%-10 \%$ of pleural effusions remain undiagnosed even after thoracoscopy. ${ }^{(15-18)}$ In one study, thoracoscopic pleural biopsy did not reveal any specific diagnosis for 9 of the 35 (25.7\%) patients with pleural effusions; these nine cases were classified as idiopathic pleural effusions. ${ }^{(8)}$ In the present study, a total of $5(12.2 \%)$ patients remained undiagnosed; of these five patients, four had undergone thoracoscopy. Although thoracoscopy was not directly diagnostic in these four patients, it was still useful, as it helped to exclude TB. The exclusion of TB, together with the absence of recurrence and stable clinical condition during follow-up at four months, pointed toward a benign aetiology, which was reassuring to the patients. Four of these five patients (one died) remain on regular follow-up.

In the present study, thoracoscopy failed in three patients due to technical difficulty. The diagnosis for two of these three patients was eventually established via bronchoscopy, while one patient remained undiagnosed. In these three patients, the thoracoscope could not be introduced into the pleural space due to prior drainage of pleural effusion, which resulted in lung expansion and apposition to the chest wall. MT is typically performed in an endoscopy suite without endotracheal intubation. Some pleural effusions should be present in the pleural space to allow for easy introduction and manoeuvrability of the thoracoscope in the pleural space. The presence of adequate pleural space (at least $6-10 \mathrm{~cm}$ in diameter) is an absolute prerequisite 
for thoracoscopy. ${ }^{(19)}$ The pre-existing pleural effusion and pneumothorax (induced by the escape of air from the atmosphere into the pleural cavity through the incision site) help to keep the lung collapsed in the pleural space, aiding in the visualisation and manoeuvring of the scope for biopsies. This is in contrast to video-assisted thoracoscopic surgery (VATS), in which single-lung intubation is used to keep the lung on the affected side collapsed to allow good visualisation and manoeuvrability of instruments in the pleural cavity.

Requirements for successful pleurodesis include even distribution of the sclerosing agent over all pleural surfaces and expansion of the lung to the chest wall. Hence, TTP (i.e. insufflation of dry talc) is recommended over the use of talc slurry. In the present study, the success rate of TTP was $77.8 \%$ at 30 days after the procedure and $80.0 \%$ at 90 days after the procedure. The success rates have been reported to be around $72 \%-90 \%$ in other studies. ${ }^{(20-24)}$ The largest study, conducted by Cardillo et al, involved 611 patients undergoing talc pleurodesis via VATS for malignant pleural effusion; in that study, the success rate was $92 \%$ over a median period of 64 months. ${ }^{(21)}$ The higher success rate in Cardillo et al's study ${ }^{(21)}$ compared with the present study could be due to several reasons. Firstly, Cardillo et al's study used VATS, which permits more pleural interventions than MT. Secondly, of the 611 patients in Cardillo et al's study, 491 (80.4\%) had a history of previous malignancy, 42 patients developed pleural effusion following surgical treatment of lung neoplasm and 482 patients were on chemotherapy. In contrast, most of our patients were newly diagnosed (only three had a history of cancer); none of our patients had undergone surgical resection of lung and only one patient was on treatment (tyrosine kinase inhibitor). Also, surgical resections performed in Cardillo et al's study, either by virtue of cure or due to less advanced stage, may have improved the success and survival rates. Additionally, $29(4.7 \%)$ of the patients in Cardillo et al's study had trapped lung and underwent decortication to enable better re-expansion. However, the $20 \%$ of patients who had trapped lung in our study did not undergo decortication, as this procedure could not be performed via MT.

The risk factors for unsuccessful pleurodesis include previous thoracic irradiation and chest tube duration of more than ten days. Pleurodesis may also fail if there is uneven distribution of agents, if the lung cannot re-expand following fluid removal, or if there is a large tumour burden with low pleural $\mathrm{pH}^{(25)}$ In the present study, the three patients who had unsuccessful pleurodesis had a median $\mathrm{pH}$ level of 7.85 (range 7.34-7.85) and a median chest tube duration of 4.5 days. No relationship was found between unsuccessful pleurodesis and fluid $\mathrm{pH}$ or chest tube duration in our study. High tumour burden of the pleural surface (e.g. multiple nodules detected on all aspects of the parietal and visceral pleura, and adherence of the lobes among themselves and with the parietal pleura) was described in the thoracoscopy reports in all three cases of unsuccessful pleurodesis. All three patients failed to have complete re-expansion of the lung after TTP. Prior to pleurodesis, one of the three patients had undergone thoracic irradiation, which has shown to be associated with pleurodesis failure. This patient presented a few days after pleurodesis with a pleurocutaneous fistula resulting in leakage of fluid through the opening in the chest wall, presumably due to poor healing of the thoracostomy wound caused by skin and subcutaneous changes from past radiotherapy. A stoma bag was subsequently applied to the site of the wound.

In the present study, $6(40.0 \%)$ of the 15 patients who underwent TTP died within one month of pleurodesis (median 16.5 [range 3-27] days). Five of these patients, who died from rapid progression of their end-stage malignant disease, should not have undergone TTP. This is because reduced survival from a poor prognosis defeats the utility of talc pleurodesis, as the patients are not able to enjoy the benefits for a prolonged period due to limited survival. Hence, TTP should only be recommended to patients whose life expectancy is more than 30 days. A Karnofsky score of 40 or higher has been shown to predict poor survival. ${ }^{(22)}$ In the present study, the decision to perform TTP was based only on the criterion of entrapped lung as visualised at the time of thoracoscopy. The use of the Karnofsky score to guide planning of TTP would have been prudent, as the procedure is best deferred in patients with low scores. The aforementioned observation highlights that there is a need for better indicators of life expectancy of less than 30 days. In the present study, response to pleurodesis did not predict survival at 30 days $(p=0.8)$ or 90 days $(p=0.73)$, similar to that shown in other studies, ${ }^{(26)}$ and the median survival duration of patients with breast cancer tended to be higher than those with lung cancer, although the difference was not significant $(p=0.31)$.

The present study was limited in that it was a retrospective study conducted in a single centre with a small sample size. However, as most of our findings are consistent with that of previous studies, they serve to strengthen the existing evidence.

In conclusion, MT with TTP is a safe and effective procedure for patients with undiagnosed effusion and those who need pleurodesis to minimise recurrence. Lack of visible pleural abnormality may predict negative biopsy. In most patients, TTP is able to provide successful and lasting palliation of symptoms that arise from recurrent pleural effusion. High tumour burden and lack of re-expansion of lung after drainage predicts failure of TTP. The development of improved therapies that increase life expectancy and the establishment of better criteria to predict that a patient has life expectancy of less than 30 days may help to improve the utility and effectiveness of TTP.

\section{REFERENCES}

1. Loddenkemper R. Thoracoscopy--state of the art. Eur Respir J 1998; 11:213-21.

2. Poe RH, Israel RH, Utell MJ, et al. Sensitivity, specificity, and predictive values of closed pleural biopsy. Arch Intern Med 1984; 144:325-8.

3. Ryan CJ, Rodgers RF, Unni KK, Hepper NG. The outcome of patients with pleural effusion of indeterminate cause at thoracotomy. Mayo Clin Proc $1981 ; 56: 145-9$.

4. Boutin C, Viallat JR, Cargnino P, Farisse P. Thoracoscopy in malignant pleural effusions. Am Rev Respir Dis 1981; 124:588-92.

5. Shaw P, Agarwal R. Pleurodesis for malignant pleural effusions. Cochrane Database Syst Rev 2004; CD002916.

6. American Thoracic Society. Management of malignant pleural effusions. Am J Respir Crit Care Med 2000; 162:1987-2001. 
7. Dresler CM, Olak J, Herndon JE 2nd, et al. Phase III intergroup study of talc poudrage vs talc slurry sclerosis for malignant pleural effusion. Chest 2005; 127:909-15.

8. Mootha VK, Agarwal R, Singh N, et al. Medical thoracoscopy for undiagnosed pleural effusions: experience from a tertiary care hospital in north India. Indian J Chest Dis Allied Sci 2011; 53:21-4.

9. Kendall SW, Bryan AJ, Large SR, Wells FC. Pleural effusions: is thoracoscopy a reliable investigation? A retrospective review. Respir Med 1992; 86:437-40.

10. Tscheikuna J, Silairatana S, Sangkeaw S, Nana A. Outcome of medical thoracoscopy. J Med Assoc Thai 2009; 92 suppl 2:S19-23.

11. Lee P, Hsu A, Lo C, Colt HG. Prospective evaluation of flex-rigid pleuroscopy for indeterminate pleural effusion: accuracy, safety and outcome. Respirology 2007; 12:881-6.

12. Wu M, Wang B, Gil J, et al. p63 and TTF-1 immunostaining. A useful marker panel for distinguishing small cell carcinoma of lung from poorly differentiated squamous cell carcinoma of lung. Am J Clin Pathol 2003; 119:696-702.

13. Ryzewicz M, McLoughlin HA, Freudenberger C, Williams A, Lindeque B. Unusual metastases from extremity soft tissue sarcomas. Orthopedics 2008 31:439.

14. Meyer PC. Metastatic carcinoma of the pleura. Thorax 1966; 21:437-43.

15. Boutin C, Viallat JR, Cargnino P, Farisse P. Thoracoscopy in malignant pleural effusions. Am Rev Respir Dis 1981; 124:588-92.

16. Oldenburg FA Jr, Newhouse MT. Thoracoscopy. A safe, accurate diagnostic procedure using the rigid thoracoscope and local anesthesia. Chest 1979; 75:45-50.
17. Menzies R, Charbonneau M. Thoracoscopy for the diagnosis of pleural disease. Ann Intern Med 1991; 114:271-6.

18. Cantó A, Blasco E, Casillas M, et al. Thoracoscopy in the diagnosis of pleural effusions. Thorax 1977; 32:550-4.

19. Janssen JP, Boutin C. Extended thoracoscopy: a biopsy method to be used in case of pleural adhesions. Eur Respir J 1992; 5:763-6.

20. Lee YC, Baumann MH, Maskell NA, et al. Pleurodesis practice for malignant pleural effusions in five English-speaking countries: survey of pulmonologists. Chest 2003; 124:2229-38.

21. Cardillo GF, Facciolo F, Carbone L, et al. Long-term follow-up of videoassisted talc pleurodesis in malignant recurrent pleural effusions. Eur J Cardiothorac Surg 2002; 21:302-5

22. de Campos JR, Vargas FS, de Campos Werebe E, et al. Thoracoscopy talc poudrage: a 15-year experience. Chest 2001; 119:801-6.

23. Diacon $\mathrm{AH}$, Wyser $\mathrm{C}$, Bolliger $\mathrm{CT}$, et al. Prospective randomized comparison of thoracoscopic talc poudrage under local anesthesia versus bleomycin instillation for pleurodesis in malignant pleural effusions. Am J Respir Crit Care Med 2000; 162:1445-9.

24. Viallat JR, Rey F, Astoul P, Boutin C. Thoracoscopic talc poudrage pleurodesis for malignant effusions. A review of 360 cases. Chest 1996; 110:1387-93.

25. Rodríguez-Panadero F, López-Mejías J. Low glucose and $\mathrm{pH}$ levels in malignant pleural effusions. Diagnostic significance and prognostic value in respect to pleurodesis. Am Rev Respir Dis 1989; 139:663-7.

26. Love D, White D, Kiroff G. Thoracoscopic talc pleurodesis for malignant pleural effusion. ANZ J Surg 2003; 73:19-22. 